Paleo-area Method

\section{Silicate Weathering}

Length

Width

Yield

Yield/2

Carbon uptake

Carbon uptake

Carbon uptake

Organic Carbon Burial

Area
yield

$$
\text { POC yield }
$$

OC burial flux

less $20 \%$ oxidized
$600 \mathrm{~km}$

$67 \mathrm{~km}$

$40200 \mathrm{~km}^{\wedge} 2$

$6.4110^{\wedge} 6 \mathrm{~mol} / \mathrm{km} 2 / \mathrm{yr}$

$3.2110^{\wedge} 6 \mathrm{~mol} / \mathrm{km} 2 / \mathrm{yr}$

$12884110^{\wedge} 6 \mathrm{~mol} / \mathrm{yr}$

$1.3 \mathrm{E}+11 \mathrm{~mol} / \mathrm{yr}$

$0.13 \mathrm{Emol} / \mathrm{m} . \mathrm{y}$

\section{$40200 \mathrm{~km}^{\wedge} 2$}

100 tons $/ \mathrm{km} 2 / \mathrm{yr}$

$100000000 \mathrm{~g} / \mathrm{km} 2 / \mathrm{yr}$

$12 \mathrm{~g} / \mathrm{mol} \mathrm{C}$

$8333333 \mathrm{~mol} / \mathrm{km} 2 / \mathrm{yr}$

$3.4 \mathrm{E}+11 \mathrm{~mol} / \mathrm{yr}$

$0.34 \mathrm{Emol} / \mathrm{m} . \mathrm{y}$.

$0.27 \mathrm{Emol} / \mathrm{m} . \mathrm{y}$.
Java (Dessert et al., 2003)

one bicarbonate returned to atmosphere per divalent cation

New Zealand (Lyons et al., 2002)

(Berner, 1982) 


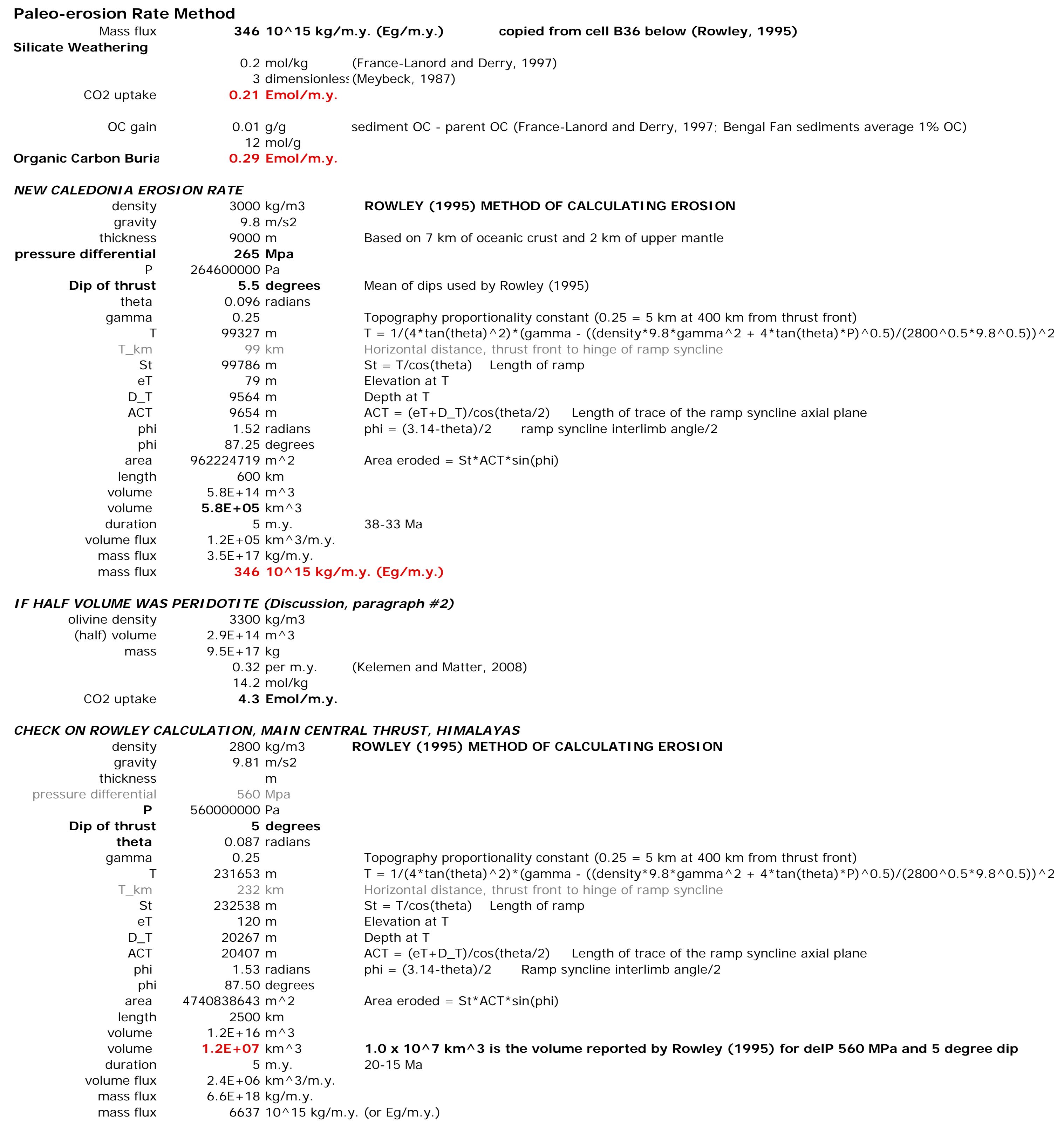

COMPARI SON WITH OTHER APPROACHES TO ESTIMATING VOLUME ERODED

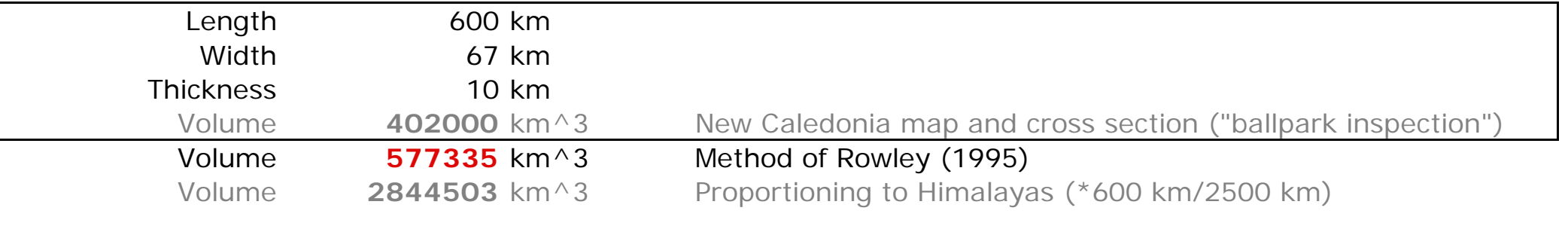




\section{Paleo-sedimentation Rate Method}

(Cluzel et al., 2001)

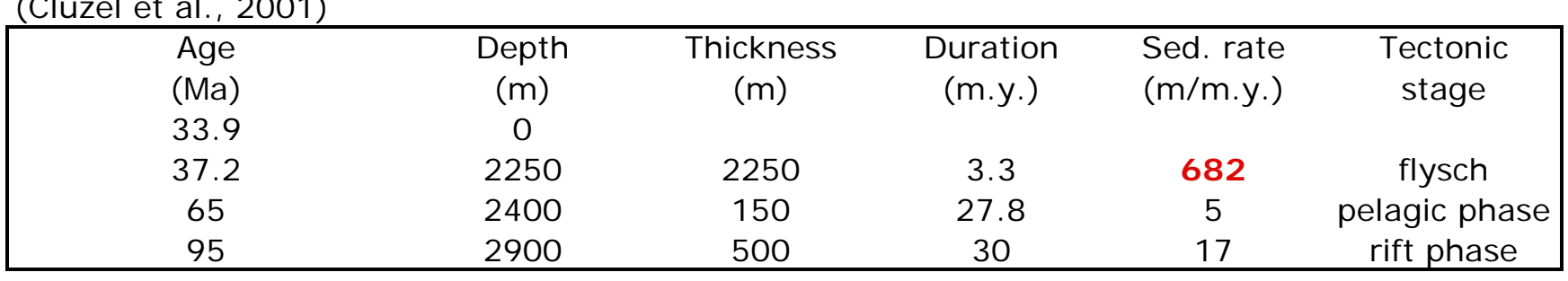

Length
NNCB
NC
LB
Total width
Area
Sed. Rate
Volume flux
Density
Mass flux
Mass flux

$800 \mathrm{~km}$

Map ( $+100 \mathrm{~km}$ at ends)

$70 \mathrm{~km} \quad$ width of N. New Caledonia Basin (Collot et al., 2008)

$30 \mathrm{~km}$

shortening on NC (Cluzel et al., 2001)

$100 \mathrm{~km}$

$200 \mathrm{~km}^{\wedge} 2$

$160000 \mathrm{~km}^{\wedge} 3$

$0.682 \mathrm{~km} / \mathrm{m} . \mathrm{y}$

$109091 \mathrm{~km}^{\wedge} 3 / \mathrm{m} . \mathrm{y}$.

$210^{\wedge} 15 \mathrm{~g} / \mathrm{km} 3$

$2.18 \mathrm{E}+20 \mathrm{~g} / \mathrm{m} \cdot \mathrm{y}$.

$218 \mathrm{Eg} / \mathrm{m} . \mathrm{y}$.

Silicate Weathering

CO2 uptake

$0.2 \mathrm{~mol} / \mathrm{kg}$

(France-Lanord and Derry, 1997)

3 dimensionless (Meybeck, 1987)

OC gain $0.13 \mathrm{Emol} / \mathrm{m} . \mathrm{y}$.

$0.01 \mathrm{~g} / \mathrm{g}$

sediment OC - parent OC (France-Lanord and Derry, 1997; Bengal Fan sediments average 1\% OC)

Organic Carbon Burial

$0.18 \mathrm{Emol} / \mathrm{m} . \mathrm{y}$ 
NOTE: Values in table are linked to calculations on previous 3 sheets

\begin{tabular}{ccccc}
\multicolumn{5}{c}{ TABLE 1. ESTIMATED CARBON FLUXES } \\
\hline \hline Method & $\begin{array}{c}\text { Silicate weathering } \\
\left(\text { Emol m.y. }{ }^{-1}\right)\end{array}$ & $\begin{array}{c}\text { Organic C burial } \\
\left(\text { Emol m.y. }^{-1}\right)\end{array}$ & $\begin{array}{c}\text { Volcanic CO2 } \\
\left(\text { Emol m.y. }^{-1}\right)\end{array}$ & $\begin{array}{c}\text { Totals } \\
\left(\text { Emol m.y. }^{-1}\right)\end{array}$ \\
\hline Paleo-area $^{\mathrm{a}}$ & 0.13 & 0.27 & --- & \\
Erosion Rate $^{\mathrm{b}}$ & 0.21 & 0.29 & --- & \\
Sedimentation Rate $^{c}$ & 0.13 & 0.18 & --- & \\
& --- & --- & 0.03 & 0.3 \\
\hline minimum & 0.13 & 0.18 & 0 & 0.5 \\
\hline maximum & 0.21 & 0.29 & 0.032 &
\end{tabular}

a) based on modern yield measurements

b) sediment-parent difference; erosion rate by method of Rowley (1995)

c) same as b), but sedimentation rate data from Cluzel et al. (2001) 
Relationship between total carbon and pCO2 (SEE GRAPH ON NEXT SHEET)

Spreadsheet from Albarede for his Figure 9.3 (Geochemistry, Albarede, 2009)

$$
\mathrm{Ks} /[\mathrm{Ca}]
$$

4.70E-05

$$
\text { 1.00E-06 }
$$

$\mathrm{H}$

K2

7.70E- 10

$\mathrm{SCO} 2$

alpha

$1.00 \mathrm{E}-07$

7.94E- 08

6. $31 \mathrm{E}-08$

5. $01 \mathrm{E}-08$

3.98E-08

3.16E-08

2.51E-08

2.00E-08

$1.58 \mathrm{E}-08$

1.26E-08

$1.00 \mathrm{E}-08$

$7.94 \mathrm{E}-09$

6.31E-09

5.01E-09

$3.98 \mathrm{E}-09$

$\mathrm{mol} / \mathrm{kg}$

2.84E-02
PCO2

$\begin{array}{ll}0.0068 & 0.021 \\ 0.0053 & 0.014\end{array}$

atm

$0.0041 \quad 0.009$

$0.0033 \quad 0.005$

0.0026

0.0020

0.003

0.0016

0.002

0.0013

0.001

0.0010

0.001

0.0008

0.001

0.0007

0.000

0.0005

0.000

$0.0004 \quad 0.000$

0.0004

0.000

0.0003

0.000

$\begin{array}{cc}8.5 & 3.16 \mathrm{E}-09 \\ 1.4 \mathrm{E}+21 & \end{array}$

0.0002

0.000

Tot C
(Emol)
9.47
7.39
5.80
4.56
3.60
2.85
2.27
1.80
1.44
1.16
0.93
0.75
0.61
0.50
0.41
0.34

PCO2

ppmv

21493

13561
8556

5399

3406

2149

1356

856

540

341

215

136

86

54

Volume of seawater (liters)

$$
1.4 \mathrm{E}+21
$$

Zachos and Kump (2005)

$\begin{array}{cc}\text { Ctot } & \text { PCO2 } \\ \text { Emol } & \text { ppmv } \\ 1 & 19 \\ 1.2 & 28 \\ 1.4 & 38 \\ 1.6 & 50 \\ 1.8 & 63 \\ 2 & 78\end{array}$

\section{THIS PAPER}

$\begin{array}{cc}\text { Ctot } & \text { PCO2 } \\ \text { Emol } & \text { ppmv } \\ 1 & 39 \\ 1.2 & 56 \\ 1.4 & 76 \\ 1.6 & 99 \\ 1.8 & 126 \\ 2 & 155\end{array}$




$\begin{array}{cccc}2.2 & 94 & 2.2 & 188 \\ 2.4 & 112 & 2.4 & 223 \\ 2.6 & 131 & 2.6 & 262 \\ 2.8 & 152 & 2.8 & 304 \\ 3 & 175 & 3 & 349 \\ 3.2 & 199 & 3.2 & 397 \\ 3.4 & 224 & 3.4 & 448 \\ 3.6 & 251 & 3.6 & 502 \\ 3.8 & 280 & 3.8 & 559 \\ 4 & 310 & 4 & 620 \\ 4.2 & 342 & 4.2 & 683 \\ 4.4 & 375 & 4.4 & 750 \\ 4.6 & 410 & 4.6 & 820 \\ 4.8 & 447 & 4.8 & 893 \\ 5 & 485 & 5 & 968 \\ 5.2 & 524 & 5.2 & 1048\end{array}$


pCO2 - total C relationship

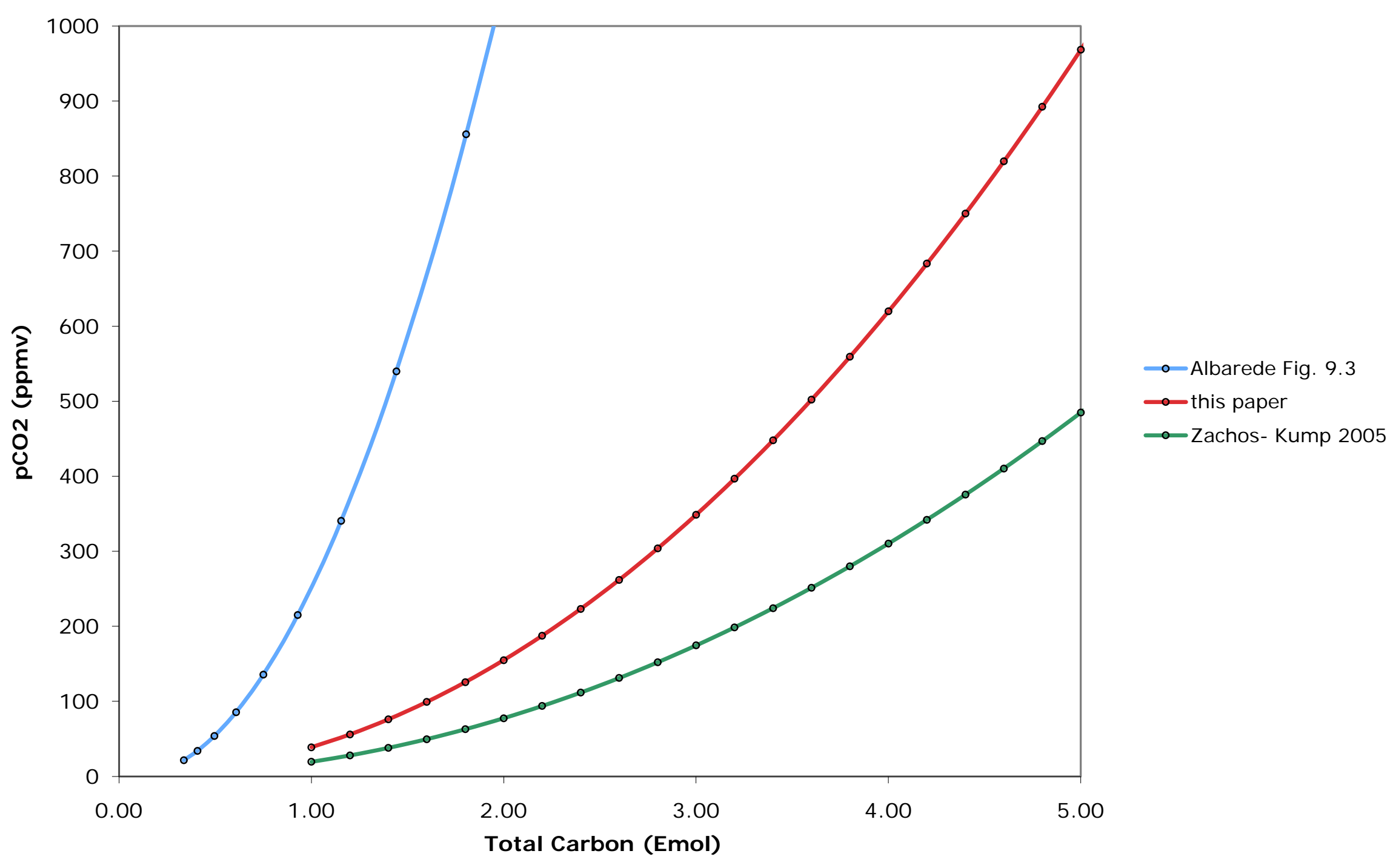


Sample integration to solve for pCO2 (Fig. 2 model results obtained using Berkeley Madonna... ... with time step of $0.1 \mathrm{~m} . \mathrm{y}$. and Runge-Kutta 4)

\begin{tabular}{|c|c|c|c|c|}
\hline $\begin{array}{l}\text { Age } \\
(\mathrm{Ma})\end{array}$ & $\begin{array}{l}\text { Time } \\
\text { (m.y.) }\end{array}$ & $\begin{array}{c}-\mathrm{d} \text { TOC/dt } \\
\text { (Emol/m.y.) }\end{array}$ & $\begin{array}{l}\text { TOC } \\
\text { (Emol) }\end{array}$ & $\begin{array}{l}\text { pCO2 } \\
\text { (ppmv) }\end{array}$ \\
\hline 36 & 0 & 0 & 5.08 & 1000 \\
\hline 35.9 & 0.1 & 0 & 5.08 & 1000 \\
\hline 35.8 & 0.2 & 0 & 5.08 & 1000 \\
\hline 35.7 & 0.3 & 0 & 5.08 & 1000 \\
\hline 35.6 & 0.4 & 0 & 5.08 & 1000 \\
\hline 35.5 & 0.5 & 0 & 5.08 & 1000 \\
\hline 35.4 & 0.6 & 0.033 & 5.08 & 998 \\
\hline 35.3 & 0.7 & 0.067 & 5.07 & 996 \\
\hline 35.2 & 0.8 & 0.100 & 5.06 & 992 \\
\hline 35.1 & 0.9 & 0.133 & 5.05 & 987 \\
\hline 35 & 1 & 0.167 & 5.03 & 980 \\
\hline 34.9 & 1.1 & 0.200 & 5.01 & 972 \\
\hline 34.8 & 1.2 & 0.233 & 4.99 & 963 \\
\hline 34.7 & 1.3 & 0.267 & 4.96 & 953 \\
\hline 34.6 & 1.4 & 0.300 & 4.93 & 942 \\
\hline 34.5 & 1.5 & 0.333 & 4.90 & 929 \\
\hline 34.4 & 1.6 & 0.367 & 4.86 & 915 \\
\hline 34.3 & 1.7 & 0.400 & 4.82 & 900 \\
\hline 34.2 & 1.8 & 0.433 & 4.78 & 884 \\
\hline 34.1 & 1.9 & 0.467 & 4.73 & 867 \\
\hline 34 & 2 & 0.5 & 4.68 & 848 \\
\hline 33.9 & 2.1 & 0.467 & 4.63 & 832 \\
\hline 33.8 & 2.2 & 0.433 & 4.59 & 816 \\
\hline 33.7 & 2.3 & 0.400 & 4.55 & 802 \\
\hline 33.6 & 2.4 & 0.367 & 4.51 & 789 \\
\hline 33.5 & 2.5 & 0.333 & 4.48 & 778 \\
\hline 33.4 & 2.6 & 0.300 & 4.45 & 767 \\
\hline 33.3 & 2.7 & 0.267 & 4.42 & 758 \\
\hline 33.2 & 2.8 & 0.233 & 4.40 & 750 \\
\hline 33.1 & 2.9 & 0.200 & 4.38 & 743 \\
\hline
\end{tabular}




$\begin{array}{rrrrr}33 & 3 & 0.167 & 4.36 & 738 \\ 32.9 & 3.1 & 0.133 & 4.35 & 733 \\ 32.8 & 3.2 & 0.100 & 4.34 & 730 \\ 32.7 & 3.3 & 0.067 & 4.33 & 727 \\ 32.6 & 3.4 & 0.033 & 4.33 & 726 \\ 32.5 & 3.5 & 0 & 4.33 & 726 \\ 32.4 & 3.6 & 0 & 4.33 & 726 \\ 32.3 & 3.7 & 0 & 4.33 & 726 \\ 32.2 & 3.8 & 0 & 4.33 & 726 \\ 32.1 & 3.9 & 0 & 4.33 & 726 \\ 32 & 4 & 0 & 4.33 & 726\end{array}$

NOTE: $\mathrm{pCO} 2=38.74 *(\mathrm{TOC})^{\wedge} 2$ 


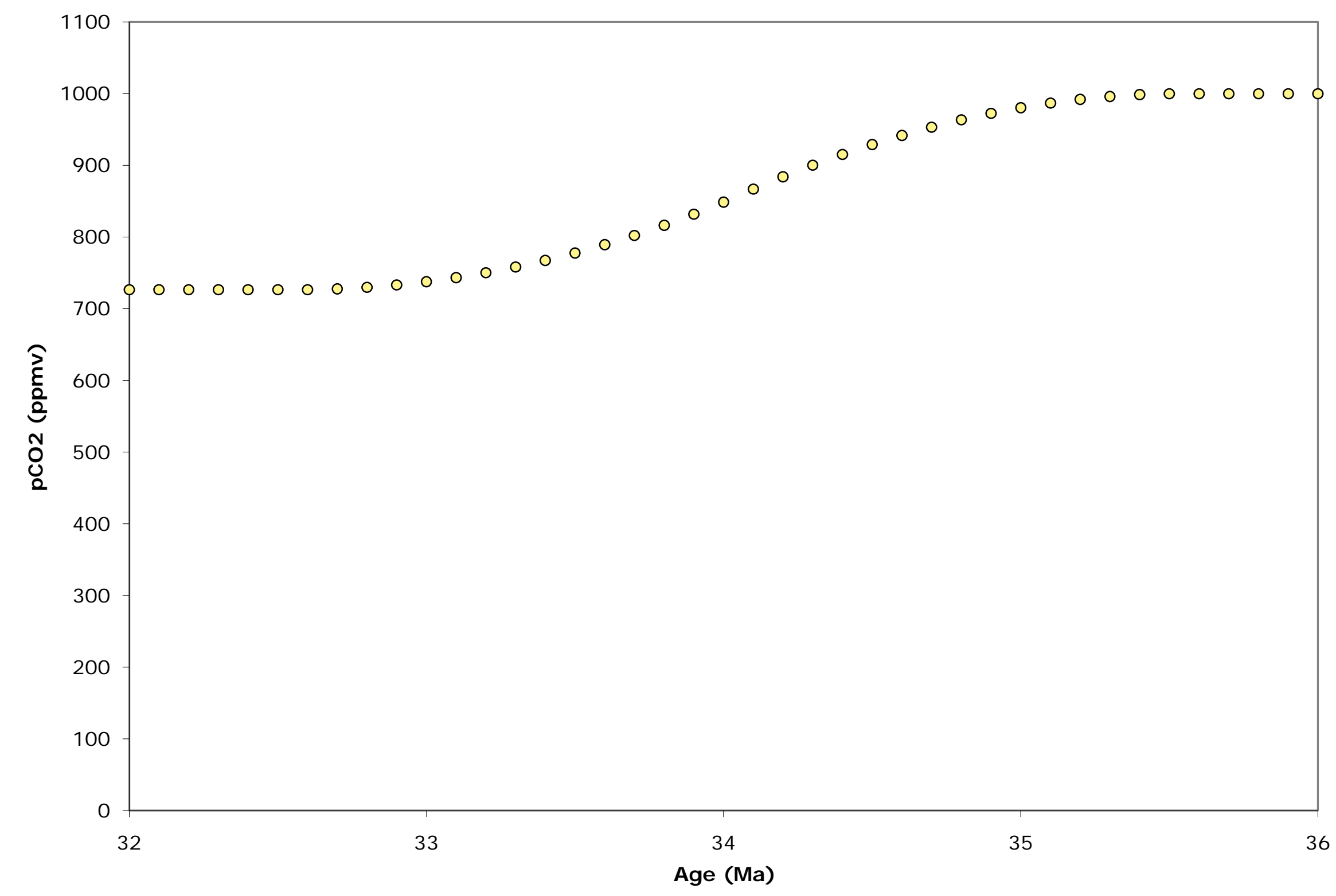




\section{Miscellaneous Calculations}

$\mathrm{kg} \mathrm{Mg}_{2} \mathrm{SiO}_{4}=>\mathrm{mol} \mathrm{C}$

$1 \mathrm{~kg}$ olivine

$1000 \mathrm{~g}$ olivine

$140.7 \mathrm{~g} / \mathrm{mol}$

$7.1 \mathrm{~mol}$ olivine

$14.2 \mathrm{~mol} \mathrm{Mg}$

\begin{tabular}{|c|c|c|}
\hline \multicolumn{3}{|c|}{ Figure 1D calculation $\left(400 \mathrm{~km}^{3} \mathrm{Mg}_{2} \mathrm{SiO}_{4}=100 \mathrm{ppmv} \mathrm{CO2}\right)$} \\
\hline Volume & $400 \mathrm{~km}^{\wedge} 3$ & \\
\hline Volume & $4 \mathrm{E}+11 \mathrm{~m}^{\wedge} 3$ & \\
\hline Density & $3300 \mathrm{~kg} / \mathrm{m} 3$ & \\
\hline Mass & $1.32 \mathrm{E}+15 \mathrm{~kg}$ olivine & \\
\hline Number & $1.88 \mathrm{E}+16 \mathrm{~mol} \mathrm{Mg}=\mathrm{C}$ & \\
\hline Mass atm & $5.00 \mathrm{E}+21 \mathrm{~g}$ & Kump \\
\hline Mean mol wt & $29 \mathrm{~g} / \mathrm{mol}$ & Kump \\
\hline Number & $1.72 \mathrm{E}+20 \mathrm{~mol}$ & \\
\hline 100 ppmv & 0.0001 & \\
\hline Number & $1.72 \mathrm{E}+16 \mathrm{~mol} \mathrm{C}$ & \\
\hline
\end{tabular}

Laboratory and Oman olivine carbonation kinetic data

$\begin{array}{cc}\text { olivine carb. } & 1 \mathrm{E}-14 / \mathrm{s} \\ & 31536000 \\ \text { olivine carb. } & 3.1536 \mathrm{E}-07 / \mathrm{yr} \\ \text { olivine carb. } & \mathbf{0 . 3 2} / \mathbf{m . y .}\end{array}$

POSSI BLE REDUCTION IN VOLCANI C DEGASSI NG WHEN SUBDUCTI ON ZONE WAS J AMMED, AND WATER DELI VERY HALTED

$1000 \mathrm{~km}$

1.6 Emol/m.y. Global arc volcanism (Hayes and Waldbauer, 2006)

$50000 \mathrm{~km} \quad$ Length of subduction zones (Bird, 2003)

2 per cent of above

$0.03 \mathrm{Emol} / \mathrm{m} . \mathrm{y}$. New Caledonian perturbation 